\title{
High Performance Oscillator Evaluation
}

\author{
J. L. Hanssen, Christopher R. Ekstrom, and Warren F. Walls \\ Time Service Department \\ U.S. Naval Observatory \\ Washington, DC 20392, USA \\ james.hanssen@usno.navy.mil
}

\begin{abstract}
We report on the results of experiments to characterizing the performance of high quality crystal oscillators. The sensitivity of the crystals to temperature and humidity were investigated. This information was used to design hermetically sealed vessels to stabilize both temperature and humidity in an effort to improve the long term frequency stability of the crystals. Preliminary data from these tests are presented.
\end{abstract}

\section{INTRODUCTION}

At the heart of many critical systems is a local oscillator that in most cases dominates the system performance at short integration times and often impacts the medium and long term performance as well. Without this stable, low noise oscillator the rest of the system would be seriously degraded or even rendered nearly useless. This is also true for devices such as hydrogen masers, atomic fountains, other passive atomic standards, and even micro-steppers. High performance crystal oscillators are typically used as the local oscillator in these systems. It is known that environmental conditions can affect the medium and long term frequency stability of crystals [1]. By reducing the impact of these outside influences through either passive or active means, it may be possible to improve the stability of these oscillators. Oscillators that are enhanced in this manner may lead to better system performance directly through their improved stability or through the ability to construct more advantageous phase locked or frequency locked loops.

In previous work [2], the effects of temperature and humidity on medium and long term stability were investigated. It was found that a change of relative humidity from $20 \%$ to $100 \%$ gave rise to a frequency shift on the order of $10^{-10}$ with a time constant of several days. It was found that by stabilizing the humidity and temperature, as well as other environmental conditions such as pressure and vibrations, improvement in long term frequency stability was achieved. They were able to reduce the level of random walk frequency noise to a level such that flicker and frequency drift were the dominant perturbations out to time scales of roughly a week.

In this paper, we present our preliminary efforts to extend this initial work. We hope to characterize any improvement to

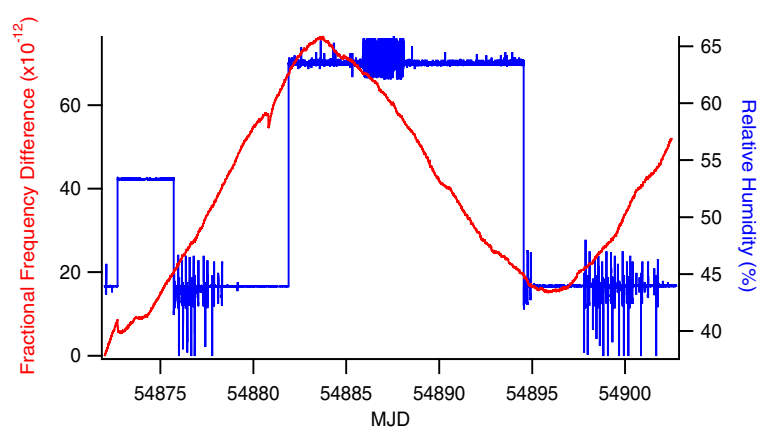

Figure 1. Figure 1: Response of crystal $\# 1$ to changes in humidity. Plotted red and read to the left is the fractional frequency difference for crystal \#1 as a function of time. Plotted in blue and read to the right is the humidity in the environmental chamber as a function of time.

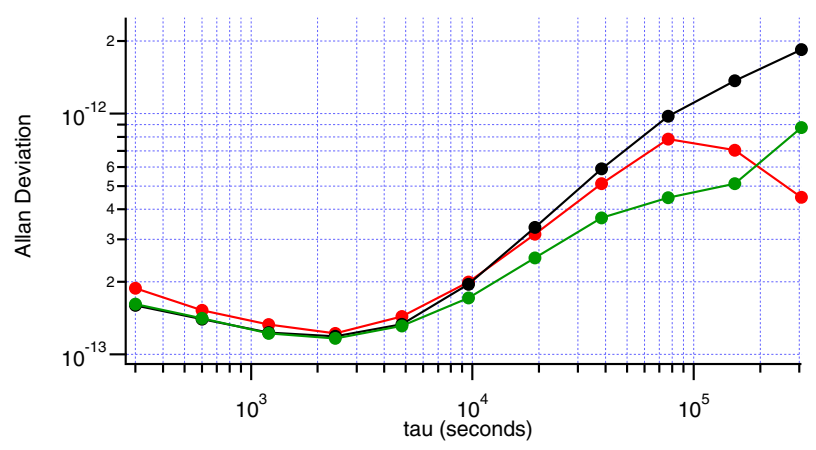

Figure 2. Frequency stability of crystal \#1. Plotted in red is the Allan deviation of crystal \#1 unsealed in an environmental chamber for a constant humidity and temperature as described in the text. Plotted in black is the frequency stability of the hermetically sealed crystal for the full data set. Plotted in green is the frequency stability for the crystal for the most recent week of data. The frequency drift has been removed for all the data sets.

humidity. By placing the oscillators in hermetically sealed boxes housed in a temperature controlled room, we have stabilized the temperature to $0.1{ }^{\circ} \mathrm{C}$ and the humidity to slow drifts of roughly $1 \%$ over one week. The outputs of these crystals have been compared to local atomic clocks which allows for good long term measurements to aid in the crystal evaluation. 


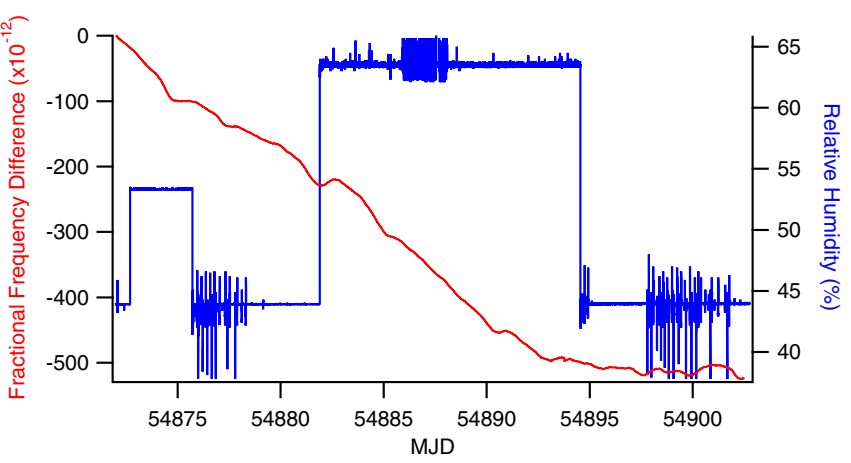

Figure 3. Response of crystal $\# 2$ to changes in humidity. Plotted red and read to the left is the fractional frequency difference for crystal \#2 as a function of time. Plotted in blue and read to the right is the humidity in the environmental chamber as a function of time.

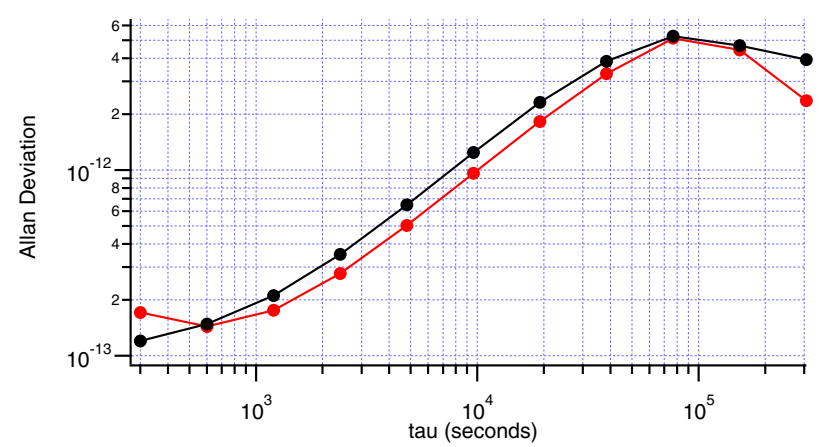

Figure 4. Frequency stability of crystal \#2. Plotted in red is the Allan deviation of crystal \#2 unsealed in an environmental chamber for a constant humidity and temperature as described in the text. Plotted in black is the frequency stability of the hermetically sealed crystal.

\section{EXPERIMENT}

Two identical high quality oscillators were used for these tests. Both oscillators are oven controlled crystal oscillators. The crystals are BVA design, SC cut, third overtone resonators producing a $5 \mathrm{MHz}$ output signal. The crystals are the same make and model with the only difference being their ages. Crystal \#1 is eight years old and has been in continual operation for seven years, while crystal \#2 is two years old with only one year of continual use. The outputs of the two crystals are measured against the USNO timing ensemble at five minute intervals.

Before performing the experiments on the environmentally stabilized oscillators, a set of baseline measurements were made of the two crystals. Both crystals were placed into a temperature and humidity controlled environmental test chamber. The temperature in the chamber was held at a fixed $23{ }^{\circ} \mathrm{C} \pm 0.25{ }^{\circ} \mathrm{C}$. The humidity was set to $44 \%$ relative humidity and the crystals were given approximately 2 weeks to acclimate to the environmental chamber. At that point, the relative humidity within the chamber was cycled through several different values and the corresponding changes in frequency output were recorded.

Fig. 1 shows the results of these tests on crystal \#1. Plotted in red is the fractional frequency of the oscillator. Plotted in blue is the humidity within the chamber. As can be

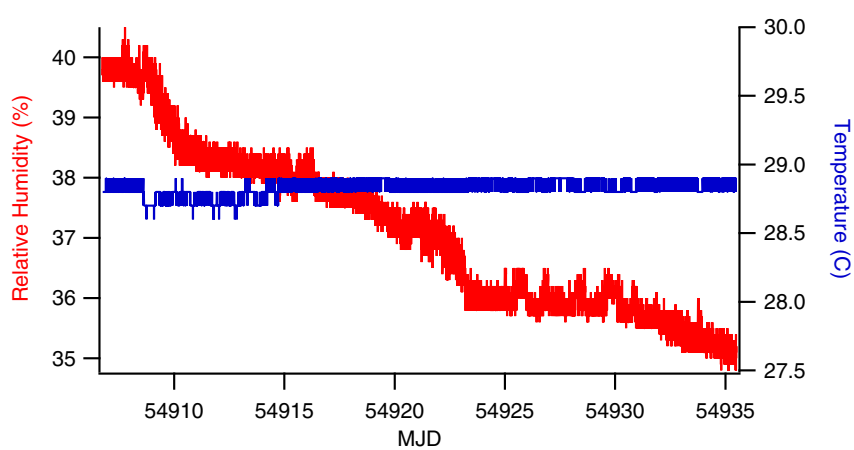

Figure 5. Humidity and temperature stability in the hermetically sealed box. Plotted in read and read to the left is the relative humidity measured inside the hermetically sealed box. Plotted in blue and red to the right is the temperature measured inside the sealed box.

seen, this crystal is sensitive to the relative humidity of its environment. At a nominal humidity of $44 \%$, the crystal has a drift rate of approximately $8 \times 10^{-12}$ per day. Upon changing the humidity to $64 \%$, the drift rate changes to approximately $6 \times 10^{-12}$ per day with a time constant of about 4 days. Unlike previous work, the drift rate did not go back to the original value but stayed at $-6 \times 10^{-12}$ per day. There was a brief (approximately 4 days) humidity setting of $54 \%$ that did not show a noticeable change in drift, but in hindsight, it is likely that the time spent at that humidity level was not sufficient to see an effect due to the long settling time.

To get a baseline stability measurement, the frequency stability within a window of constant humidity was calculated and is shown in Fig. 2. An eight day subset of the data starting four days after the humidity was set to $64 \%$ and ending before the humidity was set to $44 \%$ was chosen. The crystal shows an initial flicker floor less than $2 \times 10^{-13}$ that extends out to approximately $10^{4}$ seconds. The effects of random walk frequency noise become apparent after that with a $\tau^{1 / 2}$ dependence. A frequency drift of $-1 \times 10^{-11}$ per day was removed for this analysis. These are all within the nominal specifications of the crystal oscillator.

Crystal \#2 showed completely different behavior as can be seen if Fig. 3. Plotted in red is the fractional frequency difference of the oscillator and in blue is the relative humidity in the chamber. The crystal appears to be relatively insensitive to changes in humidity. The change of $20 \%$ in relative humidity appears not to have caused a change in drift rate. Over the majority of the test, the crystal drifts at a rate of $-2 \times 10^{-11}$ per day. Only at the end of the data set does crystal drift rate appear to change as it settles to a level of approximately $-1 \times 10^{-13}$ per day. This change is interesting, but is not correlated with the change in humidity level.

Again, the frequency stability of the oscillator was calculated for the same eight day period used for crystal \#1 when the humidity was at $64 \%$. The results of that are shown in Fig. 4. The results are similar to that of crystal \#1. It shows an initial flicker floor of less than $2 \times 10^{-13}$ that extends out to $10^{4}$ seconds after which it grows as $\tau^{1 / 2}$ which is indicative of random walk frequency noise. 


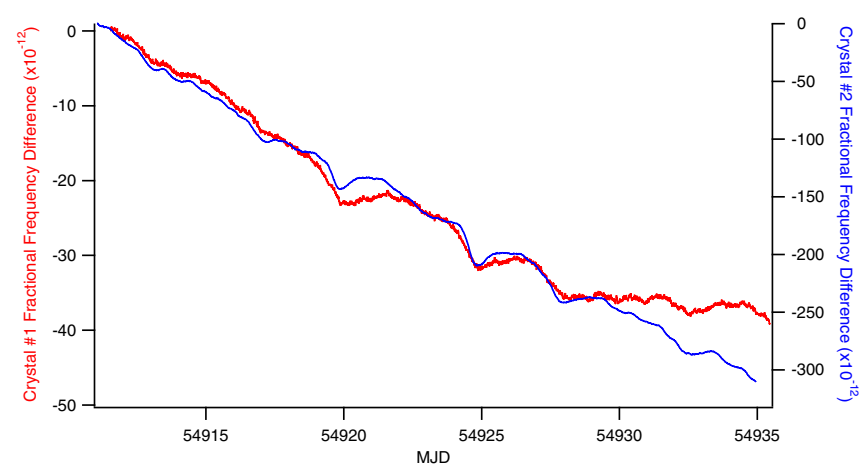

Figure 6. Crystal \#1 and crystal \#2 in hermetically sealed boxes. Plotted in red and read to the left is the fractional frequency difference for crystal \#1. Plotted in blue and read to the right is the fractional frequency difference for crystal \#2. Note that there is a different scale for the two data sets and that the data set for crystal \#2 has been delayed by 0.5 days.

The results of the baseline tests provide a good benchmark with which to compare any improvements that are brought about by stabilizing environmental conditions. It is clear that crystal \#1 is very sensitive to humidity while crystal \#2 appears to be influenced very little if at all. Therefore, stabilizing the temperature and humidity should lead to an improvement in stability for at least one of the crystals. In order to test this, the crystals were placed in hermetically sealed boxes. Two piece cast aluminum boxes were used for sealing the crystals. The two pieces had a tongue and groove sealing mechanism with a rubber gasket within the groove. Hermetic bulkhead feedthroughs were used to bring electricity into the box for the crystals and to allow the $5 \mathrm{MHz}$ signal out. After sealing the crystals within the box, weather proofing caulk was applied over all cracks and sealing points.

The temperature in the boxes is stabilized through the room temperature stabilization. The temperature in the room in which the housings were placed is stabilized to $23^{\circ} \mathrm{C} \pm$ $0.1^{\circ} \mathrm{C}$. The crystal ovens and their corresponding control electronics create a heat load within the sealed boxes that raises the temperature above the ambient room temperature by roughly $6^{\circ} \mathrm{C}$. While the ambient temperature in the box is higher than that of the room, the temperature fluctuations within the box are similar to the room temperature fluctuations of $0.1^{\circ} \mathrm{C}$

To verify that the sealing was working properly and to measure the ambient temperature and humidity that the crystals were exposed to, a small temperature and humidity sensor was placed within the box. The sensor measures relative humidity with an accuracy of $\pm 2 \%$ and temperature with an accuracy of 0.4 . The readings are recorded on a telemetry system once a minute. Fig. 5 shows the level to which the temperature and humidity were stabilized within the hermetically sealed boxes. Plotted in red is the relative humidity and in blue is the temperature in the box housing crystal \#1. Similar results were seen for crystal \#2. As can be seen, the temperature fluctuates about $28.8^{\circ} \mathrm{C}$ within a window of $0.1^{\circ} \mathrm{C}$. The humidity slowly decreases over the course of the measurements from $40 \%$ to $35 \%$. This is most likely due to a baking out process brought about by the higher ambient temperature within the sealed boxes. Over the course of the

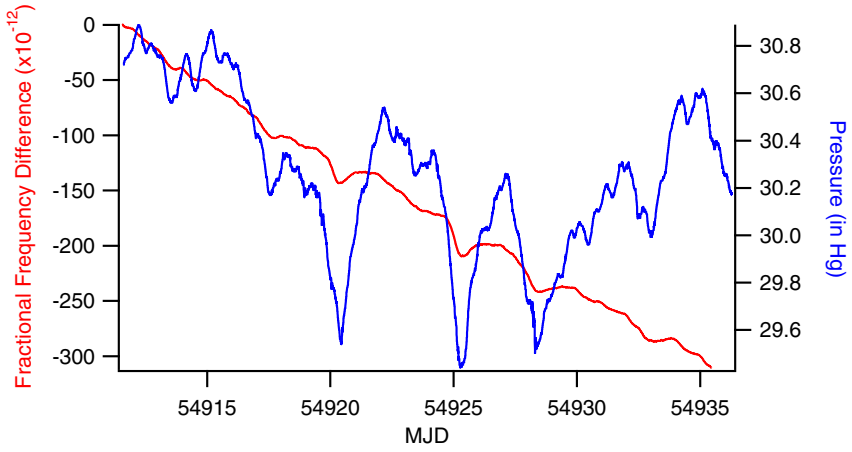

Figure 7. Comparison of frequency drifts in crystal \#2 and barometric pressure. Plotted in red and read to the left is the fractional frequency difference of crystal \#2. Plotted in blue and read to the right is the barometric pressure for Washington DC during the time of the measurements. The frequency data has been shifted to the left by 0.5 days.

data set, the relative humidity decreases at an average rate of $0.2 \%$ per day. As a testament to the seal, several times during the data set, the relative humidity in the room varied between $20 \%$ and $40 \%$ due to testing of the humidity control system in the building. These changes were never detected within the box.

Some amount of care was taken to reduce the effects of other environmental conditions. The crystals were mounted in the sealed boxes using rubber shock mounts to help vibrationally isolate them from their surroundings. As an added precaution, the housings were placed in a rack sitting atop an isolated cement pier. No additional efforts were made to minimize the effects of changing barometric pressure.

\section{RESULTS AND CONCLUSIONS}

To date, the crystals have been operating in hermetically sealed environments for 25 days. Fig. 6 shows the frequency drift of crystal \#1 over the course of the measurements plotted in red. For the first fifteen days, the crystal drifts at a rate of $2 \times 10^{-12}$ per day. Then it changes to a different drift rate of about $4 \times 10^{-13}$ per day. Fig. 2 shows the frequency stability of the crystal over the entire data set. When compared against the original baseline measurement, there appears to be no improvement.

It is interesting to look at the crystal after it changes its drift rate. The cause for the change is unknown. It is possible that the crystal has adjusted to its new environment. If that is the case and the crystal continues to behave in the same manner, then there is a definite improvement. The drift rate is excellent at $4 \times 10^{-13}$ per day. Fig. 2 shows the frequency stability over that time period which is also quite good. The Allan deviation never goes above $5 \times 10^{-13}$ for time periods corresponding to a day. More observation time is needed, but the initial results are encouraging.

Fig. 6 also shows the results for crystal \#2 which are plotted in blue. The crystal shows a drift rate of approximately $1 \times 10^{-11}$ per day which is not very different from the baseline test. It does not show any obvious change in drift rate like crystal \#1 showed. In Fig. 4, the frequency 
stability for crystal \#2 while sealed is plotted. Again, it appears that there was little improvement over the baseline tests.

Neither crystal appears to have benefitted from neither temperature nor humidity stabilization as is evident in the random walk behavior that becomes dominant at times greater than $10^{4}$ seconds. Upon inspection of the frequency data, there are distinct features that happen irregularly on the day time scale. With this in mind, it is interesting to compare the two crystals against each other. Looking at Fig. 6, it is clear that there is a common variable that is disrupting both crystals. Each dip occurs at the same place in both data sets. In addition, the relative magnitudes of the dips are the same. Please note there is a 0.5 day offset of one data set with respect to the other.

These dips appear to be due to the one environmental condition we did not specifically address: barometric pressure. Fig. 7 is an over lay of the barometric pressure for Washington D.C. with the frequency data set for crystal \#2. There is a clear correlation between the two data sets. For every low pressure system that passed through the area, a corresponding dip was seen in the frequency data. There was an immediate response in crystal \#1 to the pressure changes while there was an approximately half day lag in the response of crystal $\# 2$. The reason for the lag is unknown at this point.

Appropriately addressing the issue of barometric pressure could lead to improved long term behavior. The changes in pressure create mechanical deformations to the crystal housing that lead to frequency shifts [1]. By placing the crystals in an appropriate pressure vessel, we should be able to stabilize the pressure thereby removing some of the frequency shifts. This should give better long term frequency stability, maybe even removing the random walk frequency noise as was seen in previous work.

In conclusion, we have started to characterize the effects of stabilizing environmental conditions such as humidity and temperature on high quality crystal oscillators. We have seen that humidity can dramatically affect the drift rate of crystals, but the magnitude of the effect varies from crystal to crystal. Stabilizing the temperature and humidity of the crystal environment may have some effect, but atmospheric pressure changes eliminate any gain achieved. In future experiments, we will stabilize the pressure as well and hope to improve the long term stability of the crystals.

\section{REFERENCES}

[1] Jon Vig, "Quartz Crystal Resonators and Oscillators for Frequency Control and Timing Applications - A Tutorial," http://www.ieeeuffc.org/frequency_control/teasching.asp, Available online.

[2] Fred L. Walls, "The Influence of Pressure and Humidity on the Medium and Long-Term Frequency Stability of Quartz Oscillators," Proc. 1988 IEEE Freq. Cont. Symp., pages 279-283, 1988. 